\title{
Numerical study of thin superconducting plates subjected to transverse magnetic fields
}

\author{
Łukasz Tomków ${ }^{1,2}$ \\ ${ }^{1}$ Wrocław University of Technology, Wrocław, Poland \\ ${ }^{2}$ Joint Institute for Nuclear Research, Dubna, Russia
}

\begin{abstract}
Electromagnetic properties of the superconductors predestine them to serve as materials for the efficient and light magnetic shields. Very high attenuation factors are possible to be obtained. Superconducting elements of different geometry can be used. However, shielding effect is limited by the critical current of a superconductor.

This work describes the model of an arbitrarily shaped thin superconducting plates made of BSCCO, with the focus on circular and rectangular samples. The plates are subjected to transverse magnetic fields of different strength and shape. Changes of critical current density are considered. The distribution of the shielded magnetic field and shielding current is presented.

Results show that the magnetic field attenuation occurs and depend on the shape and material of a plate. It was also observed that coarse meshes can be used to obtain reliable results concerning magnetic field distribution, thus saving computational time.
\end{abstract}

Superconductors, magnetic shielding, magnetic fields, critical currents.

\section{INTRODUCTION}

Superconductors allow the high level of control over the shape and strength of a magnetic field. They can be used as the efficient magnetic shields, with the shielding factor of approximately $60 \mathrm{~dB}^{1}$. Superconducting tapes and bulks made of different materials were considered for such application. Gozzelino et al. analysed the behavior of $\mathrm{MgB}_{2}$ bulk samples and found that they are good passive shields ${ }^{2}$. Cavallin et al. assessed the applicability of the tubes made of the same material ${ }^{3}$. Kvitkovic et al. researched superconducting tapes made of $\mathrm{YBCO}$ and reported shielding of up to $95 \%$ of applied magnetic field ${ }^{4}$. Kim et al. characterized stacks of YBCO tape ${ }^{5}$. Wera et al. investigated the effects of geometry on shielding quality ${ }^{6}$.

The major area of applications for superconducting plates are fault current limiters, as presented by Shimizu et al, who also calculated some critical current densities ${ }^{7}$. Superconductors with different shapes can be used, as shown by Hekmati ${ }^{8}$. Kulikov et al. proposed to use superconducting magnetic shield to improve magnetic field homogeneity in an electron cooling system for a particle accelerator?.

The model created for the purpose of this paper was based on the works of Pecher et al. ${ }^{10}$, further developed by Brambilla et al. ${ }^{11}$. Grilli et al. have shown the results obtained using Comsol software $^{12}$. Hu et al. provided insight into modeling of anisotropy effects present in supercondutors ${ }^{13}$. Mohammadzadeh-Dehsorkh et al. calculated magnetization of superconducting hollow cylinder ${ }^{14}$.

\section{METHODS}

The numerical model has been developed in Comsol's PDE package. It uses H-formulation applied in $2 \mathrm{D}$. In the following equations the dimension perpendicular to the surface is marked as $z$, vertical dimension parallel to the surface is $y$ and horizontal is $x$. Since the effect of transverse magnetic field is modeled, the magnetic field $\boldsymbol{H}$ has only $z$ component, while electric field $\boldsymbol{E}$ has $x$ and $y$ components, as does current density $\boldsymbol{J}$. The main equation for the model is formula (1) based on Maxwell-Faraday equation.

$$
\mu_{0} \frac{\delta H_{z}}{\delta t}+\frac{\delta E_{y}}{\delta x}+\frac{\delta H_{x}}{\delta y}=0
$$

In this formula $t$ is time and $\mu_{0}$ is the magnetic permeability of vacuum. $\boldsymbol{J}$ is calculated as a curl of $\boldsymbol{H}$ with formula (2).

$$
\boldsymbol{J}=\nabla \times \boldsymbol{H}
$$


Electric field $\boldsymbol{E}$ in a superconducting region is found with formula (3).

$$
\boldsymbol{E}=\left\{\begin{array}{cl}
E_{0}\left(\frac{J-J_{c}}{J_{c}}\right)^{\alpha} & \text { if } J \geq J_{c} \\
0 & \text { if } J<J_{c}
\end{array}\right.
$$

$J_{c}$ is there critical current, $\alpha$ is power law exponent, assumed here as 1.5 . It is assumed that the material is isotropic. $J_{c}$ is assumed to depend on magnetic induction $\boldsymbol{B}$ according to Kim-Anderson model. The value is calculated according to the procedure described by van der Laan et al. ${ }^{15}$. $J_{c}$ consists of two paths which an electric current can take. Their individual critical current densities depend on temperature $T$ and local magnetic induction $B_{i}$. One path is between the weakly linked grains $\left(J_{c w}\right)$ and the other between strongly linked grains $\left(J_{c s}\right)$, as shown in formula (4).

$$
J_{c}\left(B_{i}, T\right)=J_{c w}\left(B_{i}, T\right)+J_{c s}\left(B_{i}, T\right) .
$$

General formula for $J_{c s}$ is shown below (equation (5)).

$$
J_{C S}\left(B_{i}, T\right)=J_{c}(0, T) \cdot \exp \left(-\frac{B_{i}}{B_{0}(T)}\right)^{\beta(T)}
$$

It is assumed that a superconducting plate is cooled with liquid nitrogen and its temperature is $77 \mathrm{~K}$. In this temperature the parameter $\beta$ is approximately 0.5 . Critical magnetic induction under zero current density $B_{0}(T)$ is calculated using formula (6). $J_{c}(0, T)$ is found with equation (6).

$$
B_{0}(T)=B_{0}(0) \cdot \exp \left(-\frac{T}{T_{s c}}\right)
$$

$T_{s c}$ is $10.7 \mathrm{~K}, B_{0}(0)$ is $107.5 \mathrm{~T}$. Therefore $B_{0}(77)$ is approximately $0.081 \mathrm{~T} . J_{c s}(0, T)$ is calculated using formula (7).

$$
J_{c S}(0, T)=J_{C S}(0,0) \cdot\left(1-\frac{T}{T_{c}}\right)^{\beta_{2}}
$$

$J_{c s}(0,0)$ is $53.4 \mathrm{~A}$, parameter $\beta_{2}$ is 0.3 and $T_{c}$ is $107.3 \mathrm{~K} . J_{c s}(77)$ is approximately $36.6 \mathrm{~A} . J_{c w}$ is calculated with formula (8).

$$
J_{c w}\left(B_{i}, T\right)=\frac{J_{c w}(0, T)}{1+\left(\frac{\left|B_{i}\right|}{B_{c w}(T)}\right)^{v}}
$$

$J_{c w}(0,77)$ is $170 \mathrm{~A}$ and $B_{c w}(77)$ is $0.001 \mathrm{~T}^{15} . v$ is assumed as 1.

Boundary conditions of the model are the values of the external magnetic field at the edges of a modeled region. A superconducting element is entirely surrounded by a normal material. The markings used to define boundary conditions and the regions of the model are shown in figure 1 . The model uses a dense regular quadrilateral mesh imposed on the superconducting region to obtain high reliability of results and a coarse triangular mesh on the normal region to reduce computational time. 


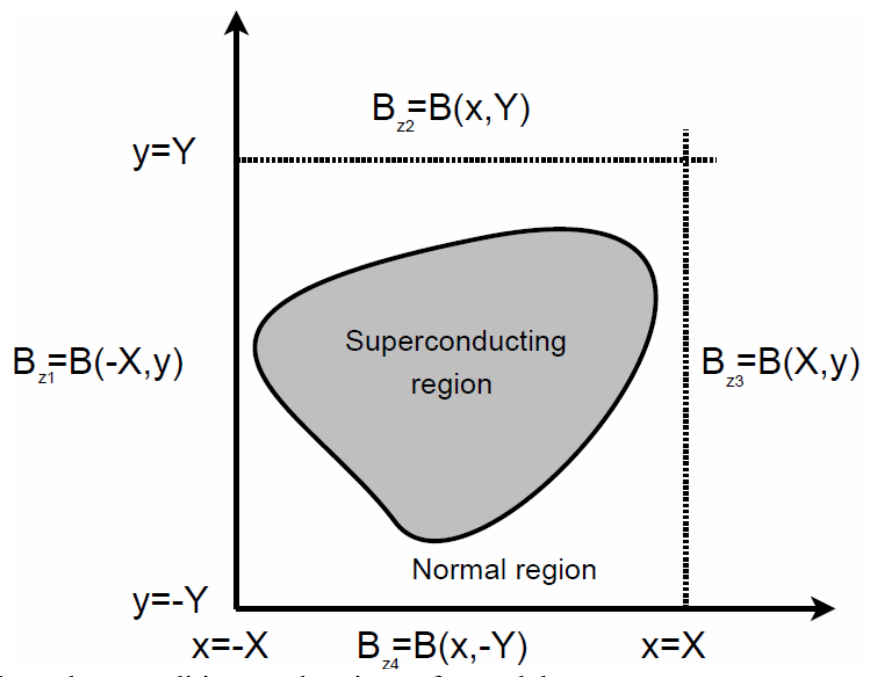

Fig. 1. Markings of boundary conditions and regions of a model

Table 1 lists the cases analyzed during simulations. In each case the values of magnetic fields from neighboring boundaries are equal at the corners to maintain stability of the model. Magnetic field applied on a boundary is calculated using equation (9). $\Gamma_{\mathrm{n}}$ is a geometrical parameter listed in table 1 and $B_{z 0}$ is the rate of field increase, assumed to be constant and equal to $0.1 \mathrm{~T} / \mathrm{s}$.

$$
B_{z n}=\Gamma_{n} \cdot t \cdot B_{z 0}
$$

\begin{tabular}{|l|l|l|l|l|}
\hline Case & $\Gamma_{1}$ & $\Gamma_{2}$ & $\Gamma_{3}$ & $\Gamma_{4}$ \\
\hline 1 & 1 & 1 & 1 & 1 \\
\hline 2 & 1 & $\sin \left(\frac{\pi x}{2 X}\right)$ & 1 & $\sin \left(\frac{\pi x}{2 X}\right)$ \\
\hline 3 & 1 & $-\cos \left(\frac{\pi x}{2 X}\right)$ & 1 & $-\cos \left(\frac{\pi x}{2 X}\right)$ \\
\hline
\end{tabular}

Table 1. Cases of boundary conditions analyzed during simulations

Differently shaped plates are considered. Distribution of magnetic field and shielding currents is found for circular and rectangular plates. Each of analyzed plates is assumed to be $1 \mathrm{~mm}$ thick.

\section{RESULTS}

Figure 2 shows how critical current density of the superconducting material depends on a local magnetic induction at the temperature of $77 \mathrm{~K}$.

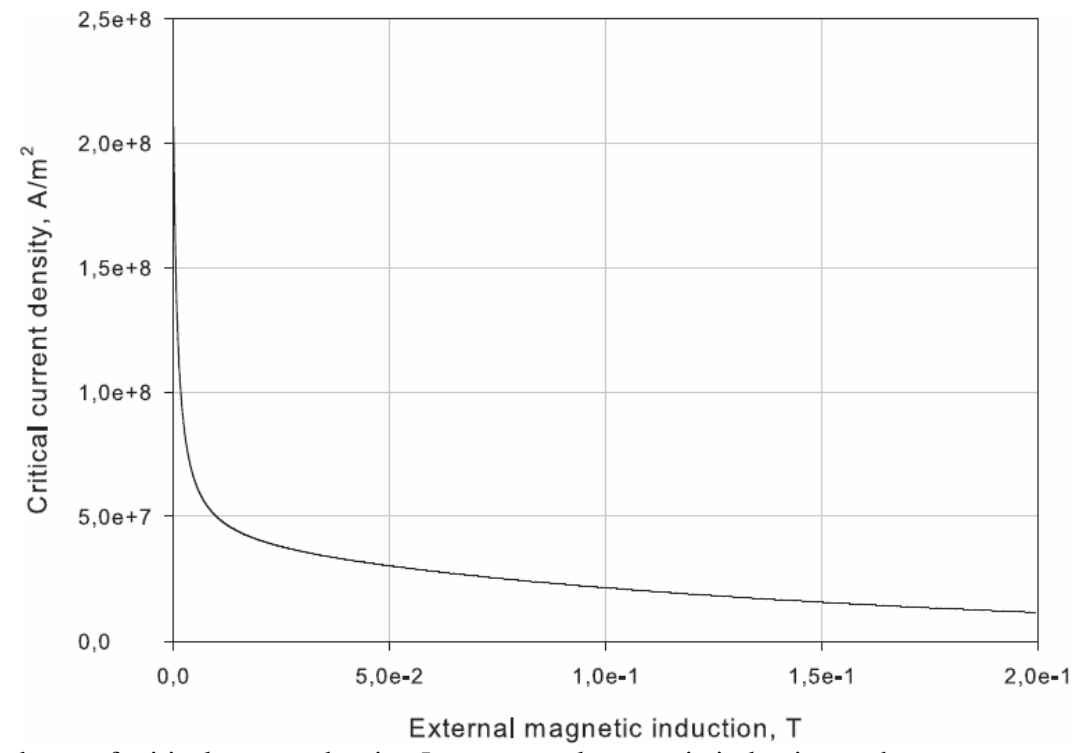

Fig 2. Dependence of critical current density $J_{c}$ on external magnetic induction at the temperature of $77 \mathrm{~K}$ 
Figures 3 and 4 show the distribution of magnetic field and shielding currents at $t=0.1 \mathrm{~s}$ for each of the analyzed cases on the rectangular and circular plates respectively. Magnetic field is given in $\mathrm{T}$, geometric dimension in $\mathrm{m}$. The length of arrows is proportional to electric current density.

The value of magnetic induction (given in T) along horizontal symmetry axis of rectangular sample is presented in figure 5. Distance from the centre of the plate given in m. Figure 6 shows electric current flowing in $1 \mathrm{~mm}$ thick fragment of the plate given in $\mathrm{A} / \mathrm{m}$ at the same symmetry axis. Both for magnetic induction and electric current the values are shown at several points in time directly corresponding to the value of applied magnetic field.

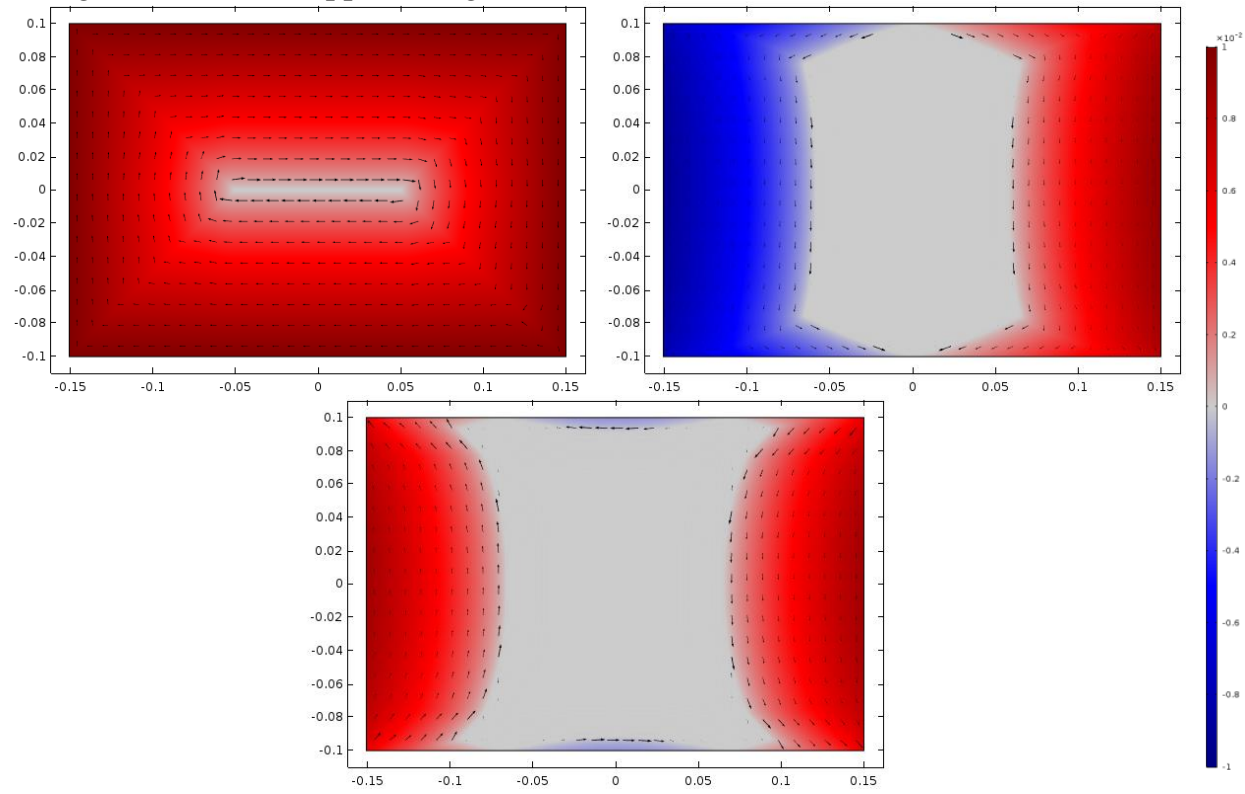

Fig. 3. Magnetic induction distribution (in T) and shielding currents on rectangular plates. From left to right, top to bottom - cases $1,2,3$

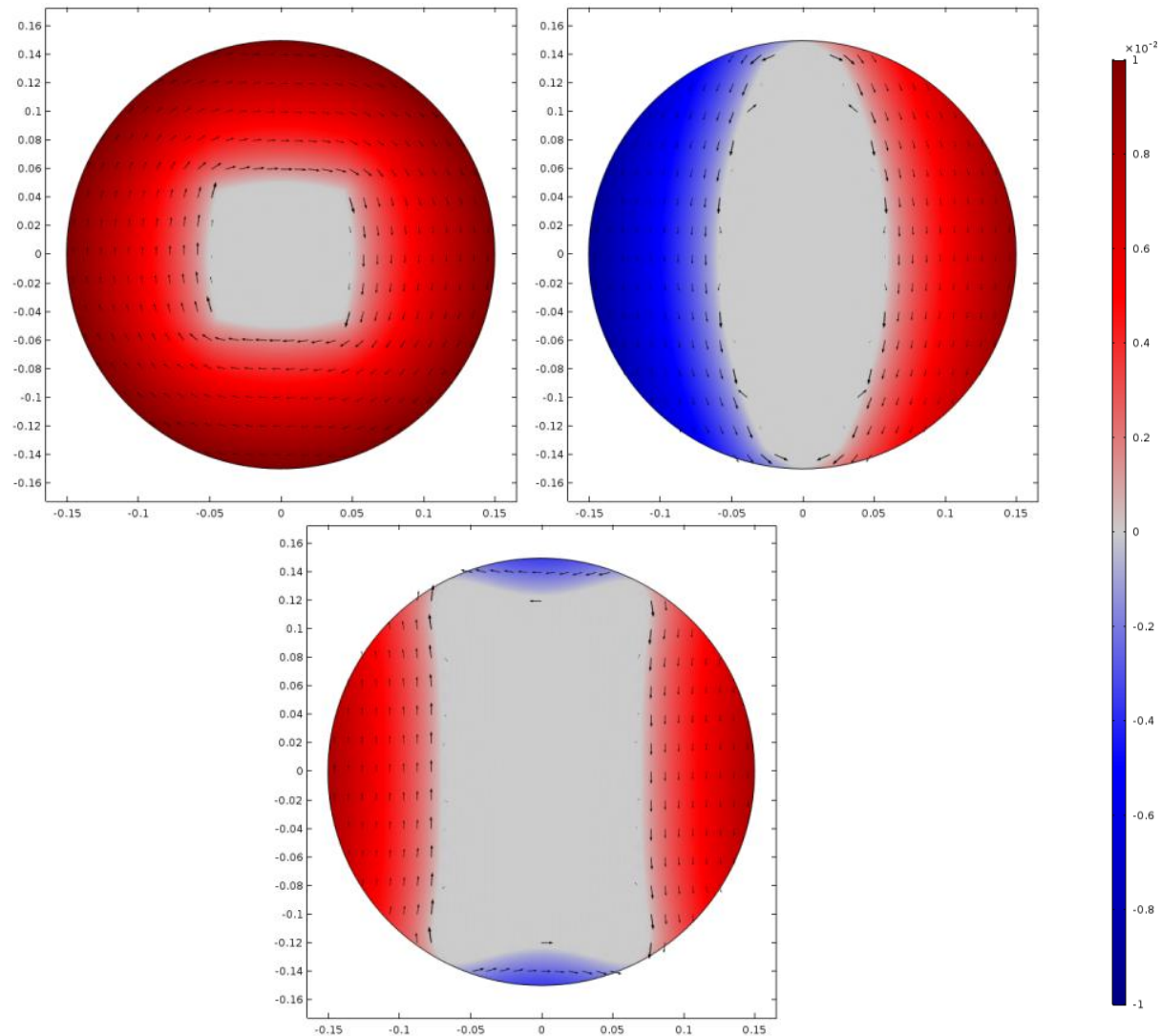

Fig. 4. Magnetic induction distribution (in $\mathrm{T}$ ) and shielding currents on circular plates. From left to right, top to bottom - cases $1,2,3$ 


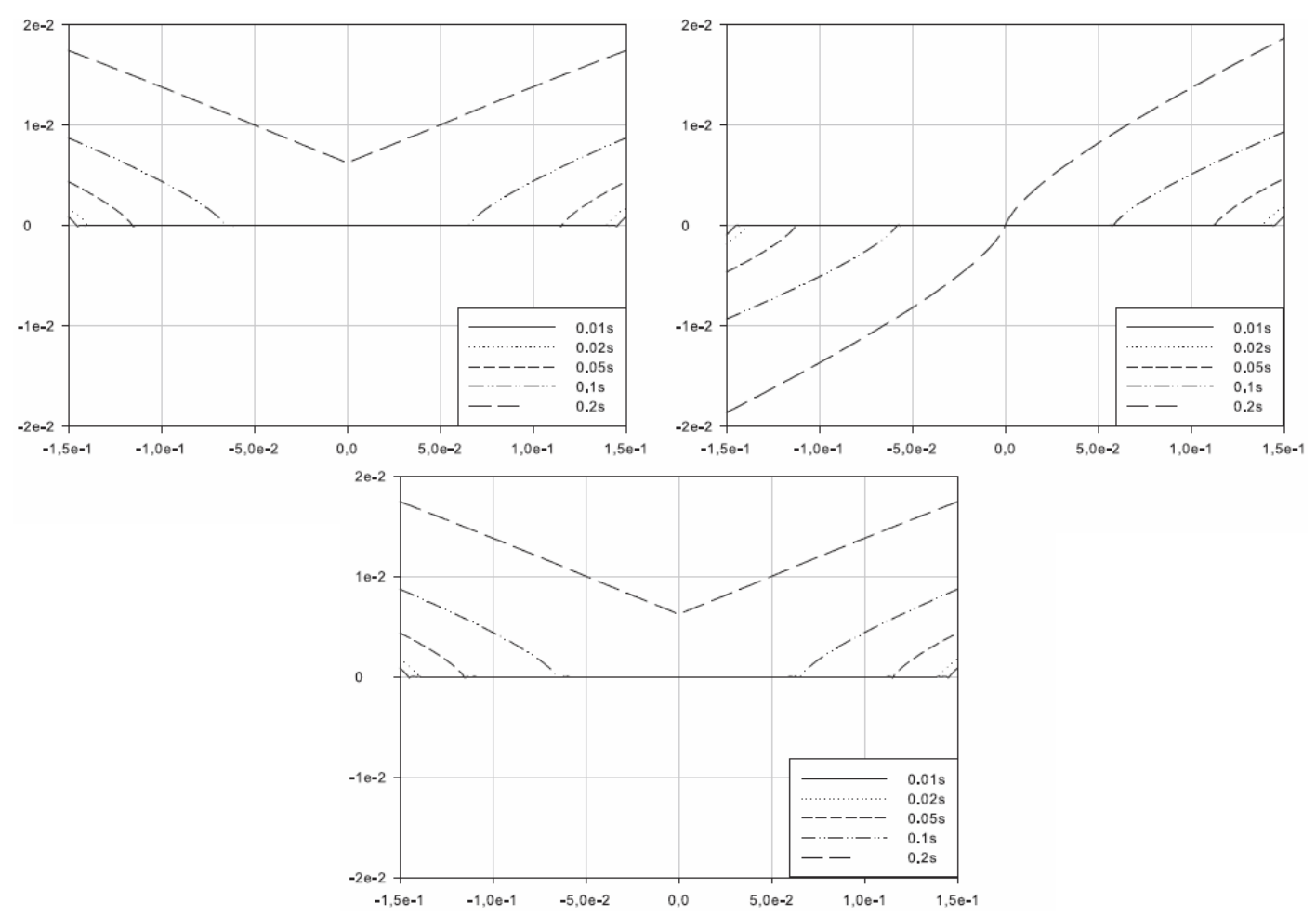

Fig. 5. Value of magnetic induction in $\mathrm{T}$ on horizontal symmetry axis of rectangular plates at different times. From left to right, top to bottom - cases 1,2,3. Geometric dimensions are given in $\mathrm{m}$.

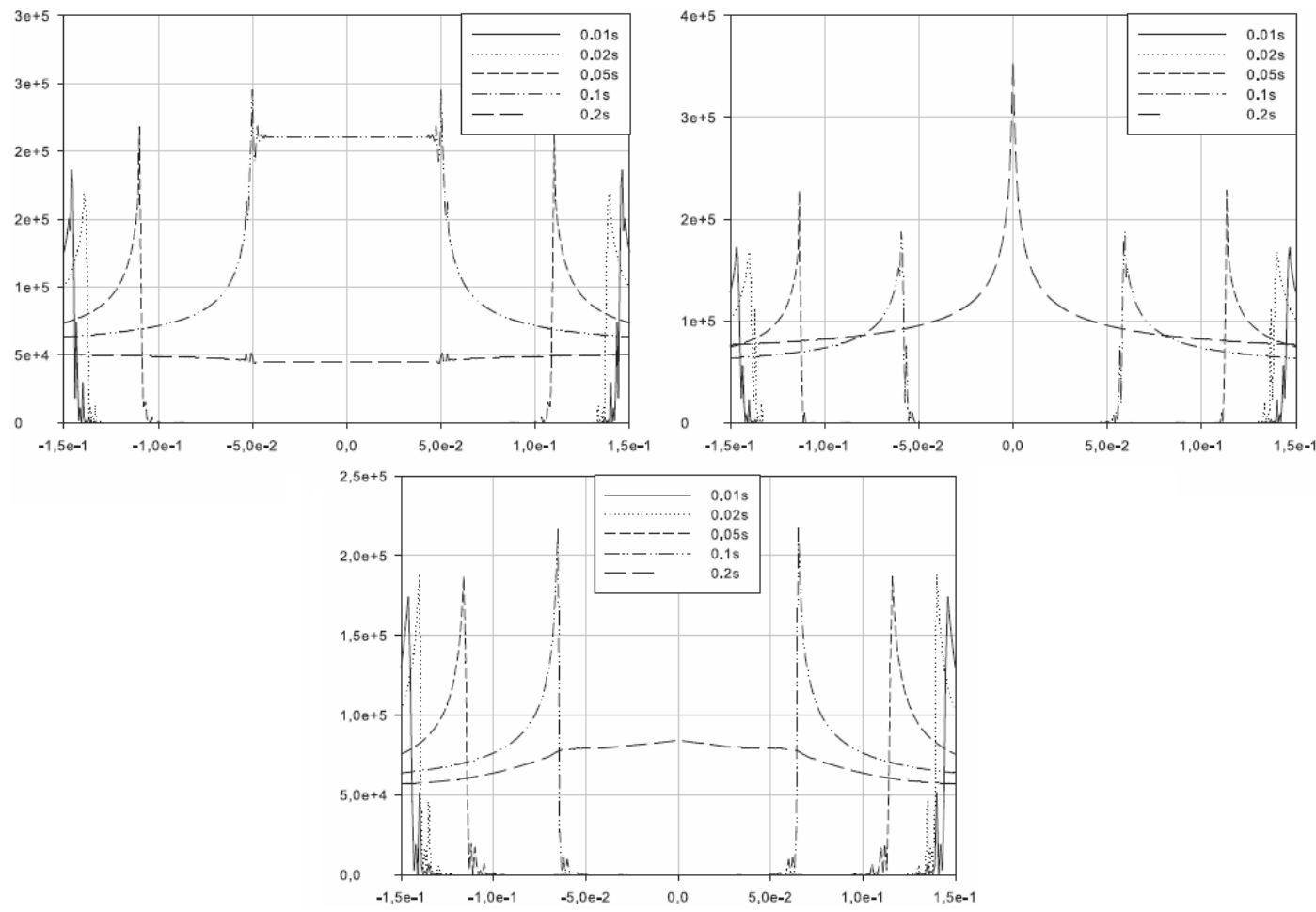

Fig. 6. Size of current density on horizontal symmetry axis of rectangular plates at different times in $\mathrm{A} / \mathrm{m}^{2}$. From left to right, top to bottom - cases 1,2,3. Geometric dimensions are given in $\mathrm{m}$.

\section{DISCUSSION}

Critical current density at the assumed temperature sharply increases when magnetic induction is below $25 \mathrm{mT}$. The maximum value of approximately $2.066 \cdot 10^{8} \mathrm{~A} / \mathrm{m}^{2}$ is obtained at $0 \mathrm{~T}$. Above this value of induction the variation is smaller and the curve flattens. At the external induction of 
$200 \mathrm{mT}$, what was the highest analyzed external induction, the critical current density is $1.1 \cdot 10^{7}$ $\mathrm{A} / \mathrm{m}^{2}$. As shown further, such shape of the curve results in specific distribution of magnetic field. Small region with zero magnetic field, known as full shielding region, can be observed in figure 3 in case 1 . Shielding currents flow around it. The distance between the edges of the plate and the region of full shielding is roughly constant. Magnetic field is slightly weaker in the vicinity of the lines connecting the corners of the plate and the region of zero magnetic field.

Pattern observed in case 2 is different. External magnetic field is weaker in the central part of the plate and the region with full shielding is larger. Similarly to case 1 the magnetic field strength is smaller on the lines connecting the corners of the plate and full shielding region, which appears concave.

In case 3 the full shielding region is becoming wider with the decrease of magnetic induction applied on the edges. It can be clearly seen that the distance over which magnetic field penetrates the plate is proportional to the strength of the applied magnetic induction. The shape is similar to cosine.

Magnetic field distribution on the circular plate, shown in figure 4, is slightly different. In case 1 a full shielding region is significantly larger, because the shielding currents have more surface to operate in vertical direction. Similarly, since the circular plate is narrower than the rectangular plate, the width of the zero magnetic field region is smaller there. Interesting effect can be observed in cases 3. The shape of shielding region which followed a pattern similar to cosine on the rectangular plate is more straight on the circular sample. This effect is caused by the interaction between the shape of the sample and the distribution of the external magnetic field.

As it can be observed in figures 5 and 6, the size and shape of magnetic induction value and electric current density on the symmetry axis of the sample change with the strength and distribution of the external magnetic field. The difference between the results obtained on considered symmetry axis of the circular and rectangular samples was small.

As shown in figure 5, the magnetic induction decreases proportionally to the local density of shielding current. In cases 1 and 3 (which are very similar) at $t=0.2 \mathrm{~s}$ the decrease is almost linear, as critical current density does not change significantly at the given induction range. In case 2 the large change of slope of magnetic induction can be seen close to the location where magnetic induction changes its direction. The width of full shielding region increases with the decrease of external magnetic induction in each case. The region disappears when the applied magnetic induction is larger than a certain threshold value.

Electric current density value presented in figure 6 generally follows the expected pattern. The spikes appear next to the beginning of full shielding region, then the current tends to disappear. Several differences can be observed in case 1 . There, at $t=0.1 \mathrm{~s}$ the current does not disappear, despite magnetic induction seems to be zero. This is probably due to the fact that the full shielding region is thinner than the resolution of a numerical mesh. Because of similar reasons some spikes are higher than the predicted critical current value.

Another effect of numerical instability can be observed at the beginning of the full shielding region. Current density slightly oscillates there. The increase of density of the mesh reduces the appearance of this effect at the cost of highly increased computation time. Reliable results for magnetic field distribution are already obtained at reasonably coarse mesh, even if the current distribution is not necessarily proper in physical sense. This is caused by the fact that, even though the value of current density in erroneous places is high, the size of such regions is very limited and the spikes appear only locally.

\section{CONCLUSIONS}

The study shows that it is possible to obtain almost complete reduction of a transverse magnetic field using thin superconducting plates. The maximum value of magnetic field which can be shielded depends on the size and material of a plate. The larger the plate the stronger the attenuation. The size and shape of a full shielding region is strongly coupled with the shape of a critical current curve. It was observed that the effect of possible numerical instabilities and errors in the distribution of current density at the borders of full shielding region weakly affects the value of magnetic field. Therefore, if the desired result is only the distribution of magnetic field, coarse meshes can be used in order for computational time to be decreased.

\section{ACKNOWLEDGEMENTS}

The Author is grateful to Wroclaw Networking and Supercomputing Center for granting access to the computing infrastructure.

The Author is grateful to Akademickie Centrum Cyfrowe Cyfronet AGH for granting access to the computing infrastructure. 


\section{REFERENCES}

[1] Denis, S., Dusoulier, L., Dirickx, M., Vanderbemden, P., Cloots, R., Ausloos, M. and Vanderheyden, B., "Magnetic shielding properties of high-temperature superconducting tubes subjected to axial fields," Superconductor Science and Technology 20(3), 192 (2007).

[2] Gozzelino, L., Minetti, R., Gerbaldo, G., Ghigo, F., Laviano, G., Lopardo, C., Plapcianu, A., Agostino, S., Cagliero, M., Truccato, L., Zilberti, L. and Mezzetti, E., "Magnetic Characterization of $\mathrm{MgB}_{2}$ Bulk Superconductor for Magnetic Field Mitigation Solutions," Journal of Superconductivity and Novel Magnetism 24(1-2), 307 (2011).

[3] Cavallin, T., Quarantiello, R., Matrone, A. and Giunchi, G., "Magnetic shielding of $\mathrm{MgB}_{2}$ tubes in applied DC and AC field," Phys.: Conf. Ser. 43, 1015 (2006).

[4] Kvitkovic, J., Pamidi, S. and Voccio, J., "Shielding AC magnetic fields using commercial $\mathrm{YBa}_{2} \mathrm{Cu}_{3} \mathrm{O}_{7}$-coated conductor tapes," Superconductor Science and Technology 22(12), 3577 (2009).

[5] Kim, J., Kim, C., Patil, P., Kvitkovic, J. and Pamidi, S., "Electrical Characteristics of Stacks of YBCO Tapes in Applied Magnetic Field," IEEE Transactions on Applied Superconductivity 21, 3230 (2011).

[6] Wéra, L., Fagnard, J., Levin, M., Vanderheyden, G. and Vanderbemden, P., "Magnetic Shielding With YBCO Coated Conductors: Influence of the Geometry on Its Performances," IEEE Transactions on Applied Superconductivity 23(3), 8200504 (2013).

[7] Shimizu, H., Sannomiya, N., Watanabe, Y., Yokomizu, T., Matsumura, M., Shibuya, M., Ichikawa, H. and Kado, H, "Theoretical analysis of current density distribution in superconducting plate of flat type superconducting fault current limiter," Journal of Physics: Conference Series 43, 933 (2006).

[8] Hekmati, A., "Modeling of Shield-Type Superconducting Fault-Current-Limiter Operation Considering Flux Pinning Effect on Flux and Supercurrent Density in High-Temperature Superconductor Cylinders," Journal of Superconductivity and Novel Magnetism 27(3), 701 (2014).

[9] Kulikov, E., Agapov, N., Drobin, V., Smirnov, A., Trubnikov, G., Dorofeev, G. and Malinowski, H., "Improving homogeneity of the magnetic field by a high-temperature superconducting shield," Journal of Physics: Conference Series 507, 032028 (2014).

[10] Pecher, R., McCulloch, S., Chapman, L. and Prigozhin, L., "3D-modelling of bulk type-II superconductors using unconstrained H-formulation," Proc. EUCAS 2003 (2003).

[11] Brambilla, R., Grilli, F. and Martini, M., "Development of an edge-element model for

AC loss computation of high-temperature superconductors," Supercond. Sci. Technol. 20, 16 (2007).

[12] Grilli, F., Sirois, F., Laforest, M. and Ashworth, S., "Space-time Formulation for FiniteElement Modeling of Superconductors," Proceedings of the COMSOL Conference 2008 (2008).

[13] Hu, D., Ainslie, M., Zou, J. and Cardwell, D., "Numerical Analysis of Non-Uniformities and Anisotropy in High-Temperature Superconducting Coils," IEEE Transactions on Applied Superconductivity 25(3), 4900605 (2015).

[14] Mohammadzadeh-Dehsorkh, B. and Babaei-Brojeny, B.,"Magnetization in a Finite Superconducting Hollow Cylinder in the Presence of the Radial and Azimuthal Transport Currents," Journal of Superconductivity and Novel Magnetism 28(6), 1711 (2015).

[15] van der Laan, D., van Eck, H., ten Haken, B., Schwartz, J. and ten Kate, H., "Temperature and magnetic field dependence of the critical current of $\mathrm{Bi}_{2} \mathrm{Sr}_{2} \mathrm{Ca}_{2} \mathrm{Cu}_{3} \mathrm{O}_{\mathrm{X}}$ tape conductors," IEEE Transactions on Applied Superconductivity (1), 3345 (2001). 